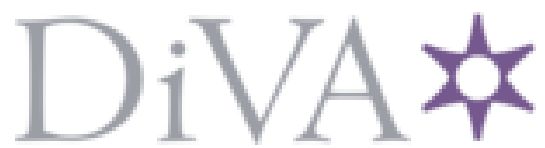

http://www.diva-portal.org

This is the published version of a paper presented at 14th International Conference The Experience of Designing and Application of CAD Systems in Microelectronics (CADSM).

Citation for the original published paper:

Kuzminykh, I., Snihurov, A., Carlsson, A. (2017)

Testing of communication range in ZigBee technology

In: 14th International Conference The Experience of Designing and Application of CAD Systems in Microelectronics (CADSM), 21-25 Feb. 2017, Lviv, Ukraine Lviv, Ukraine: Institute of Electrical and Electronics Engineers (IEEE) https://doi.org/10.1109/CADSM.2017.7916102

N.B. When citing this work, cite the original published paper.

Permanent link to this version:

http://urn.kb.se/resolve?urn=urn:nbn:se:bth-15951 


\title{
Testing of Communication Range in ZigBee Technology
}

\author{
Ievgeniia Kuzminykh ${ }^{1}$, Arkadii Snihurov ${ }^{1}$, Anders Carlsson ${ }^{2}$ \\ 1. Telecommunication Systems Dept., Kharkiv National University of Radio Electronics, UKRAINE, Kharkiv, Nauki av. 14., email: \\ yevheniia,kuzminykh@nure.ua,arkadii.snihurov@nure.ua \\ 2. Computer and Communication Systems Dept, Blekinge Institute of Technology, SWEDEN, 37179, Karlskrona, BTH Campus Gräsvik, \\ email: anders.carlsson@bth.se
}

Abstract: In the rapidly growing Internet of Things (IoT) applications from personal electronics to industrial machines and sensors are getting wirelessly connected to the Internet. Many well-known communication technologies such as WiFi, ZigBee, Bluetooth and cellular are used for transfer data in IoT. The choice of corresponding technology or combination of technologies depends on application or other factors such as data requirements, communication range, security and power demands, battery life.

In this paper we will focuse on ZigBee wireless technology and testing ZigBee end devices in order to see how transmission range impacts on quality parameters.

Keywords: ZigBee, XBee, IoT, X-CTU, transmission range.

\section{INTRODUCTION}

Internet of Things conception consists of majority of connected devices such as microcontrollers, sensors, other wireless devices, actuators that provide the fingers, brain and eyes of the Internet of Things. All these devices are belong to "last inch" of the network. The aim is not allow users to connect to all these end devices simultaneously. Problems of realization of applications related to IoT devices are quite different from those associated with traditional network end points.

Since only a small amount of data typically must be transmitted between these connected devices, reliable, costeffective wireless communication protocol like ZigBee is ideal. Energy efficiency is critical because these devices are often not connected to a power source, and have to be operated during several years without maintenance or battery replacement, using just the energy-collecting source as solar panels or its embedded single battery. Developers also need to take into account factors such as cost, system size, microcontroller characteristics, component count, standards, compatibility, security factor, ease-of-use and troubleshooting of such devices. Finally, the software is required to connect all devices, aggregated sensor data and to provide information to end users in secure way through Internet displaying information on their displays, computers, tablets or smartphones.

ZigBee technology [1] is used to build various "smart home" systems: lighting control, air-conditioning and heating, alarm sensors and appliances and so on. In most cases, ZigBee networks are deployed indoors but manufacturers indicate the operating range of transceivers in open space, that is why it is difficult to tell what the communication range would be in real conditions. For this purpose studies have been conducted to determine how obstructions influence the communication range inside a building, as well as how buildings influence communication range in open space.

In this paper we will test efficiency of ZigBee end devices in room and on open air.

\section{ZigBeE TECHNOLOGY}

Widespread use of wireless technology ZigBee is caused by quick applicability for building wireless sensor networks with low transmission rate, up to $250 \mathrm{kbit} / \mathrm{s}$. ZigBee as Bluetooth technology has a huge pre-installed base of operation, but perhaps more frequently in industrial settings. ZigBee PRO and ZigBee Remote Control (RF4CE), among other available ZigBee profiles, are based on the IEEE 802.15.4 [2] protocol, which is an industry-standard wireless networking technology operating at $2.4 \mathrm{GHz}$ targeting applications that require relatively infrequent data exchanges at low data-rates over a restricted area and within a $100 \mathrm{~m}$ range such as in a home or building.

ZigBee Remote Control protocol has few significant advantages among systems offering low-power transmission, robustness, high security and high scalability with high node density and it also takes $\mathrm{n}$ advantage of wireless control and sensor networks in IoT applications [3-5]. ZigBee latest version is the recently launched v. 3.0 which is essentially the combination of the various ZigBee wireless standards in a single standard. An example product and kit for ZigBee development are TI's CC2538SF53RTQT ZigBee SystemOn-Chip IC and CC2538 ZigBee Development Kit.

\section{ANALYSIS OF ZIGBEE MODULES}

The main objective which was set for the experiment is to determine the actual communication range when using XBee wireless modules for both indoor and outdoor communication, in urban areas, as well as to define practical recommendations for their use.

To conduct analysis of the communication range between two ZigBee modules we need:

1) Two XBees modules - XBees exist in a variety of series, frequencies, and ranges.

2) Two adaptors - either the Adaptor USB, Adaptor USB Dongle, or Adaptor Serial. These boards act as an 
interface between your computer and an XBee. They're used to configure your $\mathrm{XBee}$ and pass data to and from your computer.

Depending on which explorer you have, you may also need a matching mini-B USB or serial cables.

3) At least one computer with X-CTU [6] installed. The latest version of X-CTU is available for both Mac and Windows [7].

XBee and XBee-PRO Modules were made for ZigBee/IEEE 802.15.4 standards and support such requirments for wireless sensor networks as low-cost and low-power. Modules need minimal power and provide reliable delivery of critical data between devices.

The modules operate with $2.4 \mathrm{GHz}$ frequency band and are pin-for-pin compatible with each other.

Currently available in the territory of Ukraine are transceivers known as XBee modules manufactured by Digi (MaxStream).

For testing we used XBee module series 1 XB24 - A (Fig.1).

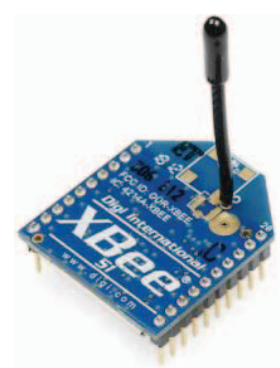

Fig.1. The XBee module used in the experiment

Characteristics of modules:

- Indoor/Urban: up to $30 \mathrm{~m}$;

- $\quad$ Outdoor line-of-sight: up to $100 \mathrm{~m}$;

- Transmit Power: $1 \mathrm{~mW}(0 \mathrm{dBm})$;

- Receiver Sensitivity: $-92 \mathrm{dBm}$;

- $\quad$ RF Data Rate: 250,000 bps.

Advanced Networking and Security is provided by:

- Point-to-point, point-to-multipoint and peer-to-peer topologies supported;

- Retries and acknowledgements;

- 128-bit encryption;

- Direct Sequence Spread Spectrum (DSSS);

- Each direct sequence channels has over 65000 unique network addresses available;

- Self-routing or Self-healing mesh networking.

Feathers of XBee modules:

1) Low Power:

- $\quad$ RX Current: $50 \mathrm{~mA}(3.3 \mathrm{~V})$;

- $\quad$ TX Current: $45 \mathrm{~mA}(3.3 \mathrm{~V})$;

- Power-down Current: $<10 \mu \mathrm{A}$.

2) Easy-to-Use :

- Software is free (X-CTU);

- no configuration is necessary for out-of box RF communications;

- AT Command Mode, configuration of module parameters is simple;

- Small form factor;

- Network compatible with other 802.15.4 devices.
3) Free and unlimited technical support.

There are two modules XBee and XBee-PRO.

$\mathrm{XBee}$ Series 1. These modules are simple to use, as they do not require any configuration to operate in peer to peer communication. That means you can directly replace a wired serial connection with these devices.

XBee Series 2. These modules are slightly complex to use as they require some configuration even to work in peer to peer communication. If you are just starting with XBee and your requirement is just replace a serial wired connection it is recommended you work with Series 1, though Series 2 is more power efficient than Series 1.

\section{Components Setup}

The first stage of setting up any network is to ensure the necessary components are configured correctly, this in turn will ensure the accuracy of the data collected during the investigation and analysis of the network. ZigBee network is not too complicated to setup and run as there are components to configure.

Connect the mini USB cable to the adaptor module. The USB interface board is a "plug-and-play" device that should be detected by the PC automatically. To interface between the modem and a PC, two drivers must be installed: a USB driver and a virtual COM port driver that makes the USB port look and perform like a physical COM port. After the modem is detected, a wizard for installing the USB driver is launched.

To add XBee(s) click the "Add device" tab in X-CTU menu. After select communication port or USB Serial port accordingly and configure serial characteristics like baud rate, data bits, and stop bits. Click the module, and wait a few seconds as X-CTU reads the configuration settings the XBee. The right half will contain the entire configuration of the XBee.

Two modules both are at default values but one is set as Coordinator, and the other as End Device.

\section{TESTING OF COMMUNICATION RANGE}

\section{A. Indoor Experiments}

Two indoor experiments were conducted with the first requiring the signal to be propagated through walls in rooms and the second was conducted in the corridor.

\section{A.1. Room to room}

Upon analyzing the data obtained it can be concluded that the number of packets received, regardless of the size of a transmitted packet, decreases sharply between 5-10 meters, as shown in Fig. 2.

There are the condition concerning to the experiment:

- $\quad$ at 0 meters there were no barriers;

- $\quad$ at 5 meters the barrier was a one Curtain wall;

- $\quad$ at 10 meters the barrier was one Curtain wall and one Structure wall;

at 15 meter's the barrier was two Curtain walls and one Structure wall (at which point the modules were unable to connect).

Sighting all this data, it is evident that the module requires clear line of sight for effective interaction. 


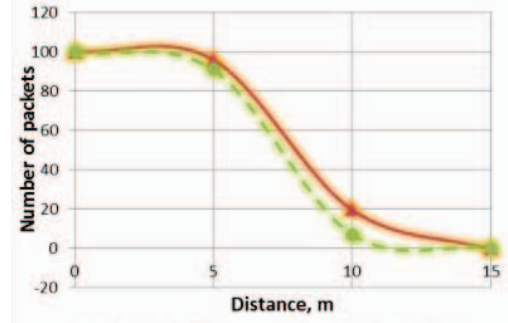

$\rightarrow$ Packet size 2 bytes - - - Packet size 32 bytes

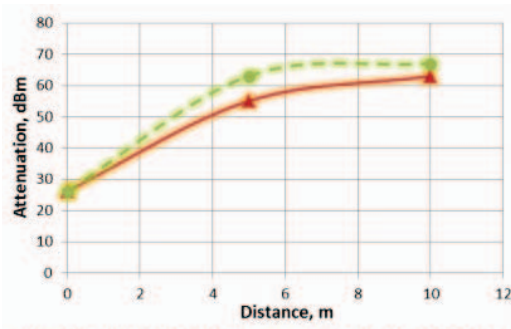

- Signal level at $R \times 2$ bytes - - - Signal level at $R \times 32$ bytes

Fig.2. Results for Room to Room Experiment

\section{A.2. Corridor Experiments}

In this case how signal reception quality with direct sight conditions depends on the distance between the modules, signal level $(\mathrm{dBm})$ at the receiver input were measured (Fig.3).

$\mathrm{X}$-CTU program allows evaluating signal level in $\mathrm{dB}$, and counting the percentage of received packets.

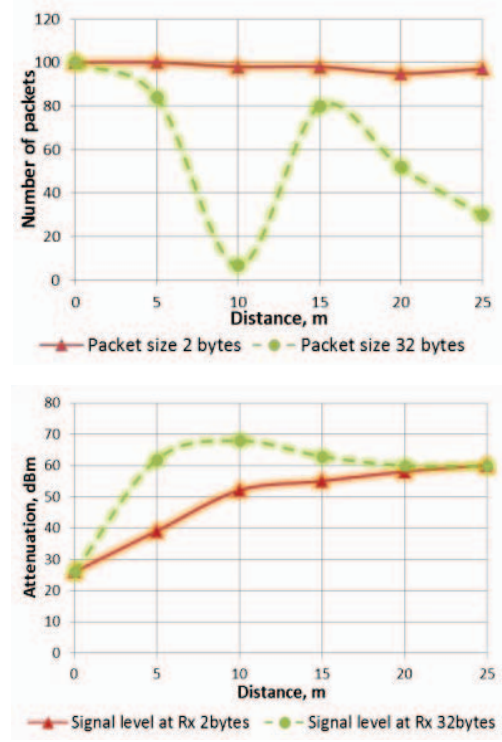

Fig.3. Results for Corridor Experiment

A closer analysis of the graph shows an anomaly in expected data for the received packet size ( 32 bytes), this is due to the fact that as the experiment was conducted in the corridor of an active university, on occasion someone would walk through the corridor thus disrupting the signal hence packet losses occur, then as line of sight is restored packets received begin to rise again.

It is also worth mentioning that the module did not reach the stipulated $30 \mathrm{~m}$ indoor range, this could be because of reflections causing significant fading of the signal or just that the module is defective.
In most practical implementations, ZigBee networks operate in enclosed areas (when the units are line-of-sight visible) but indoors. For such cases, the model of propagation of radio signals inside buildings is often applied - a model of log-normal distribution of path loss [8]:

$$
\begin{aligned}
P l(d)= & P L\left(d_{0}\right)+10 \alpha \log \left(\frac{d}{d_{0}}\right)+N\left(0, \sigma_{c h}^{2}\right) \\
& =\overline{P L}(d)+N\left(0, \sigma_{c h}^{2}\right)[d B]
\end{aligned}
$$

where $d$ is the distance between the transmitter and the receiver $(\mathrm{m})$;

$d_{0}$ - reference distance $(\mathrm{m})$;

$\alpha$ - exponent of path loss;

$N\left(0, \sigma_{c h}^{2}\right)$ Gaussian random variable with zero mean and variance $N\left(0, \sigma_{c h}^{2}\right)[\mathrm{dB}]$.

\section{B. Open Space Experiments}

For this experiment two wireless modules were used too. Experiment conditions are shown in Fig.4, the red line shows the movement of one of the modules in the experiment, the red dot shows the stationary positioned module.

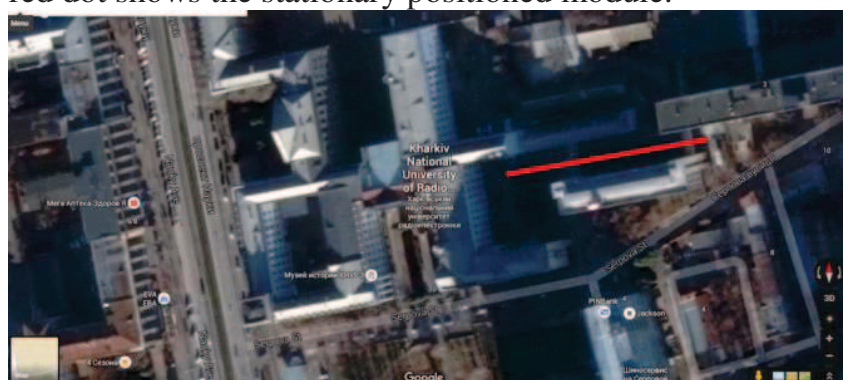

Fig.4. Outdoor Experiment

For propagation model in open space it is assumed that there are no obstacles and reflecting objects between the transmitter and the receiver [8]. The following Eq. (2) allows you to find the amount of attenuation in open space, depending on the distance between the modules:

$$
P_{l}=20 \log \left(\frac{\lambda}{4} \frac{\pi}{R}\right)
$$

where $R$ is a distance between transmitter and receiver (meters);

$\lambda$ - wavelength (in this case, $2.4 \mathrm{GHz}$ );

$P_{l}$ - loss on path of propagation of radio signal.

Open space in urban areas is not completely free of barriers as there are still obstacles like buildings; movement

\begin{tabular}{|c|c|c|c|c|c|c|c|}
\hline \multirow[b]{2}{*}{$\begin{array}{c}\text { \# of the } \\
\text { experiment }\end{array}$} & \multirow{2}{*}{$\begin{array}{c}\text { Time } \\
\text { interval } \\
\text { between } \\
\text { packets, } \\
\text { ms }\end{array}$} & \multicolumn{3}{|c|}{ Packets } & \multirow{2}{*}{$\begin{array}{l}\text { Signal leve } \\
\text { at the } \\
\text { receiver } \\
\text { input, } d B\end{array}$} & \multirow[b]{2}{*}{$\begin{array}{c}\text { Distance } \\
\text { between } \\
\text { modules, } \mathrm{m}\end{array}$} & \multirow[b]{2}{*}{$\begin{array}{l}\text { Packet } \\
\text { size, } \\
\text { byte }\end{array}$} \\
\hline & & received & lost & $\begin{array}{c}\text { Percentage of } \\
\text { received } \\
\text { pockets, } \%\end{array}$ & & & \\
\hline 1 & \multirow{12}{*}{1000} & 190 & 10 & 94,5 & -86 & 110 & \multirow{12}{*}{32} \\
\hline 2 & & 196 & 4 & 98 & -78 & 100 & \\
\hline 3 & & 200 & 0 & 100 & -76 & 90 & \\
\hline 4 & & 191 & 9 & 95,5 & -83 & 80 & \\
\hline 5 & & 171 & 29 & 85,5 & -83 & 70 & \\
\hline 6 & & 196 & 4 & 98 & -82 & 60 & \\
\hline 7 & & 200 & 0 & 100 & -82 & 50 & \\
\hline 8 & & 200 & 0 & 100 & -73 & 40 & \\
\hline 9 & & 177 & 23 & 88,5 & -79 & 30 & \\
\hline 10 & & 200 & 0 & 100 & -74 & 20 & \\
\hline 11 & & 200 & 0 & 100 & -68 & 10 & \\
\hline 12 & & 200 & 0 & 100 & -40 & 0 & \\
\hline
\end{tabular}
of cars, people, etc. The results are shown in Table 1.

\section{TABLE 1. RESUlts FOR THE MODULES IN OPEN SPACE}


Fig.5 shows how the percentage of received packets and signal attenuation depend on the distance.

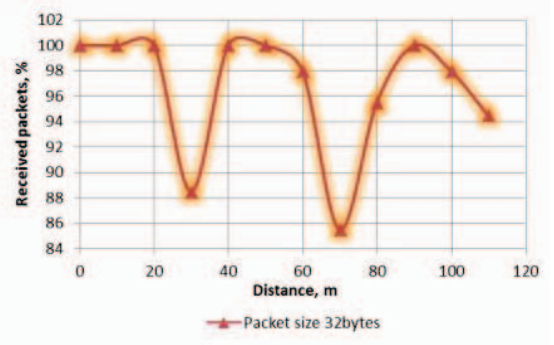

Fig.5. Results for Open Space Experiment

Analyzing the data obtained it can be concluded that the number of received packets apparently reduced, as it is seen in Fig.5, due to the presence of two passages between the university buildings. The presence of line-of-sight (direct visibility) suggests that the first Fresnel zone is completely open. In the conditions of our experiment, there is a partial closing of the first Fresnel zone with obstacles (passage), and increase of the number of reflections and time delay of radio signal, which leads to an even more significant fading of the radio signal in a receiver, to a loss of data packets and degradation of the communication channel.

The movement of people also leads to a change in the quality of communication. This is because the signals reflected from various surrounding objects (multipath reception) converge at the receiving point. At the same time, depending on the relative position of surrounding objects the signals can both be amplified and attenuated at the receiving point. The strong influence of the reflected signals is accentuated by observations during the experiment.

\section{CONCLUSION}

In this paper the configuration and testing of ZigBee modules was preformed through the use of the software $\mathrm{X}$ CTU. The program gives the user total access to the XBee module allowing the user to set parameters to their specific desire with settings such as (channel=C, PAN ID=3332, $\mathrm{DH}=0, \mathrm{DL}=0, \mathrm{MY}=0$ ). The two modules were configured to communicate as one was set as a Coordinator and the other an End Device and range tests were performed. Analysis was made of the indoor range capabilities, with two experiments conducted.

Room to room experiment was designed to investigate the modules ability to transmit through walls, the results reviled that the module requires clear line of sight for optimum transmission

Corridor and open space experiments were designed to investigate the module indoor and outdoor, line of sight range, to determine the effects the surrounding environment has on the signal. The results suggest factors such as the ones below could have influenced the signal:

- $\quad$ Scattering is the deviation of signal from the straight line when the signal hits an object whose size is in the order of wavelength or less.

- Reflection occurs when the signal hits a surface that is larger compare to the wave-length of the signal.

- Diffraction occurs when the signal is obstructed by sharp edges.
- Refraction occurs when the signal propagates through air. Refraction is the bending of the radio wave, while propagating through the atmosphere.

Having analyzed theoretical and practical results it can be concluded that the recommended distance between the units indoors is a distance not exceeding 25 meters, and for open space, taking into account possible obstacles, is 60 meters. In the presence of obvious obstacles a repeater should be used. The use of efficient data transmission route provides implementation of ZigBee technology in everyday life.

Obtained results show that during designing wireless network with sensor nodes that is base for Internet of Thing technologies you should be very careful with choice of end devices because the actual communication range for both indoor and outdoor communication may not correspond to the declared values.

\section{REFERENCES}

[1] "Zigbee Alliance", Zigbee.org, 2017. [Online]. Available: http://www.zigbee.org/

[2] "IEEE 802.15.4. IEEE Standard for Low-Rate Wireless Networks", http://standards.ieee.org/, 2017. [Online]. Available: http://standards.ieee.org/getieee802/download/802.15.42015.pdf

[3] R. Sutaria and R. Govindachari, "Making sense of interoperability: Protocols and Standardization initiatives in IOT", in The 2nd ComNeT-IoT workshop in the 14th International Conference on Distributed Computing and Networking ICDCN 2013, Mumbai, 2013.

[4] Internet of Things. IoT Governance, Privacy and Security Issues, IERC - European Research Cluster on the Internet of Things, Final Report, January, 2015. [Online]. Available: http://www.internet-of-thingsresearch.eu/pdf/IERC_Position_Paper_IoT_Governance_ Privacy_Security_Final.pdf

[5] D. Miorandi, S. Sicari, F. De Pellegrini and I. Chlamtac, "Internet of things: Vision, applications and research challenges", Ad Hoc Networks, vol. 10, no. 7, pp. 14971516, 2012.

[6] "XCTU - Next Gen Configuration Platform for XBee/RF Solutions - Digi International", Digi.com, 2017. [Online]. Available: solutions/xctu-software/xctu

[7] "Exploring XBees and XCTU - learn.sparkfun.com", Learn.sparkfun.com, 2017. [Online]. Available: https://learn.sparkfun.com/tutorials/exploring-xbees-and$\mathrm{xctu}$

[8] S.S. Baskakov. "ZigBee standard and MeshLogic Platform: routing efficiency in the "many-to-one'", The first mile, № 2, pp.32-37, 2008. 